\title{
Acceso a la producción argentina en humanidades y ciencias sociales: representatividad en bases de datos internacionales multidisciplinarias
}

\author{
SUSANA Romanosde TIRATEL \\ Instituto de InvestigacionesBibliotecológicas, FFyL, Universidad \\ de Bue nos Ai res. Puán 480, $4^{\circ}$ piso, ofi ci na 8, C1406CQJ \\ Buenos Aires, Argentina. \\ E-mail: inibi@filo.uba.ar
}

\section{RESUMEN}

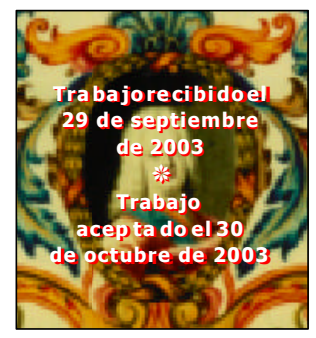

Se pre sen tan los prime ros re sul ta dos de una in ves ti ga ción que es tu dia las ta sas de representatividad, de traslapamiento y de vacancia de la producción sobreartículos deinves tiga ción es pecializados en humanida des y cien cias sociales en bases de da tosin terna cionales. Eles tu dio se en marca en elcon tex to teó rico de M. Ke resz te si, y de talla los ras gos que debe po seeruna re vis ta para ser con si de ra da aca dé mi ca. So bre esta base se par te de una lis ta ini cial de 356 títulos depublica ciones perió dicas argen tinas que se con fron taron conlas listas de publicaciones seriadas analizadas por bases de datos internacionales multidisciplinarias.Se dan las ta sas derepre sen ta tividad delos países la tinoame ri ca nos en las ba ses de da tos ele gi das, en tre ellos la Argen ti na, y la re presen ta tividaddeés tas enla producciónargentina. Secomparanlas ca rac terís ticas di fe ren cia les delos servi cios dein di za ción y re sú menes, y se ex po nen las implicaciones de los resultados alcanzados.

Pa la bras cla ve: Producción académica; Ar gen ti na; Humanidades; Ciencias sociales; Bases de datos internacionales multidisciplinarias. 


\title{
ACCESS TO THE ARGENTINIAN PRODUCTION IN HUMANITIES AND SOCIAL SCIENCES: ITS REPRESENTATION IN MULTIDISCIPLINARY INTERNATIONAL DATABASES
}

\author{
SusANa Romanosde TIRATEL
}

\begin{abstract}
The pres ent study in volves the pre limi nary re sults of a re search study ing the representativity rates of overlapping and absence in the production of spe cialized re search pa pers in Hu mani ties and So cial Sci ences in in ternational databases. The paper uses M. Keresztesi's work as theoretical frame work, and de scribes the fea tures a se rial must pos sess in or der to be con sid ered an aca demic jour nal. Ac cord ing to these cri te ria, a list of 356 Argentine titles was made and compared with the serials indexed by international databases. The rates of representation for Latin American countries in the selected databases are given, including among them those of the Argentinian production. The differential characteristics of indexing and abstracting services are compared and the implications of the results are shown.

Keywords: Aca de mic Production; Ar gen ti na; Humanities; Social Sciences; International Multidisciplinary Databases.
\end{abstract}

\section{INTRODUCCIÓN}

$\mathbf{L}$ a Argen tina ca re ce enlaactuali dad de una es truc tu rabibliográfica de ac ce soa su producción cien tí fi ca; si bien a lo lar go de su his to ria los pro yec tos que em prendió para controlar bibliográficamente la información generada por la investigación en diferentes áreas del conocimiento se caracterizaron por la discontinuidad y, la mayo ría, poruna vida efí me ra. ${ }^{1}$ Den tro de di cha pro duc ción, las hu ma ni da des y las ciencias sociales ocupan un lugarmuy im por tan te. Sin em bargo, seig no ra el co noci mien to pro ducido porunnú mero signi ficativo deinves tiga doresnosólo en el ex terior sino tam bién den tro del pro pio país. La tec no logía de la in for ma ción ha per mi ti do un desarrollo sostenido y cada vez más sofisticado de bases de datos bibliográficos que analizanlosdocumentos delmicro-mundobibliográficopublicadosmayoritariamente en los países centrales. La producción de las zonas periféricas está pobremente representada -en caso de que lo esté- y los investigadores cuentan con pocas herramientaselaboradas en otros países para co no cer lo que se pu bli ca en el pro pio. Así, la bús quedabibliográficaseconvierteenunprocedimiento de alto cos to y bajos be ne ficios, dado que para lograr una lista, no ya exhaustiva, sino al menosrepresentativa,

1 Den tro delos ejem plos más des ta ca dos se pue den ci tar laBibliografíaargentinade ArtesyLetras. № 1 (ene.-mar. 1959)-52 (jul.-dic. 1971). Bue nos Ai res: Fon do Na cio nal de las Artes, 1959-1974.52 v. y la Bibliografiaargentinade Ciencias Sociales / Fun da ción JoséMa ría Ara gón, Red de In for ma ción so bre Ciencias Sociales REDICSA. 1982-1989. Buenos Ai res: Fun da ción Ara gón, 1983-1989. 8 v. 
se deben consul tarmu chas ba ses con resul ta dos redun dan tes yes ca sos y, porlotanto, desalentadores.

Anteesta si tua ción com pe te a cada uno delos países bus carel con trol ex haus tivo delalitera turaderiva da delasactivida des deinves tigacióny constituirasílabibliografía na cionalespecializa da de cada país, en este caso, la Argentina. Alres pec to, hay que separardosgrupos biendiferenciados: (a) eldelosdocumen tospertenecientesalma cro-mun do bibliográ fico, yasea pu blica dos (libros, se ria das como un todo, publica ciones oficiales, de con gre sos y con fe ren cias, pa tentes, normas, leyes y de cre tos, etcétera), especiales (microformas, partituras, fotografías, películas, videocasetes, etcétera) onopublicados(tesis, informes, memoranda,correspondencia,etcétera); y (b) eldelos documen tos pertenecientesalmundomicro-bibliográ fico (capítulosde libros de au to res múltiples, artículos depublica cionesperió dicas, ponencias dec ongresosyconferencias, etcétera). ${ }^{2}$

Paraabordarunatarea de semejante envergadu ra sede be ráencararunapla nifica cióna es cala na cional, y para que sea ra cional yefec tiva habrá que diagnos ti car el estado de la situación actual en cada una de las categorías documentales previamente enuncia das en to daslases pecialida des cubiertas porlainves tiga ción. Den trode este con tex to se ubicala in ves tiga ción que se está realizan do en el Ins ti tu to de Inves tigaciones Bibliote cológicas delaFacultad deFilo so fíay Letras, la cual hasidoevaluada, acreditada y subsidiada por la Secretaría de Ciencia y Técnica de la Universidad de BuenosAires(UBACYTF602-Programación2001-2003.) ${ }^{3}$

El tema delapublica ción pe rió dica es com plejo, sus en foques son muy diversosy son variados los intereses asociados con la revista especializada, entre los cuales se pue den men cio nar:la pro li fe ración, la dis persión, la de mo ra enla publica ción,la diversidad de roles, la caracterización unívoca y los costos crecientes, ${ }^{4}$ por enumerar sólo al gu nos de los abor da jes po sibles. De esta si tua ción se de riva uno de los in convenientes para procesar la bibliografía latinoamericana referida a esta problemática que, a las cues tio nes an tes men cio na das, agre ga otras pro pias de la re gión: fal ta de regularidaddelaspublicacionesperiódicasatribuiblealaescasez defondospermanentes de dicados a su finan ciamien to; el ama teu ris modelos res pon sables de sucon creción; la ca ren cia de po líticas cien tí fi cas cla ras y sos te nidas en el tiem po; las fallas enla dis tribu ción y di fu sión de los títulos; la presencia casinula de títu los dela tinoamericanos en las ba ses de da tos in ter na cio na les (so bre todo enlas del Ins ti tu te forScien tific

2 Susana Roma nos de Tira tel.Guíadefuentesdeinformaciónespecializadas:Humanidadesy Ciencias Sociales. 2a. ed. Buenos Aires: GREBYD, 2000, p. 18-22.

3 Indicadoresdelaactividaddeinvestigaciónaplicadosalasrevistasargentinas Proyectodeinves tiga ciónaprobado y subsidiado por la Secretaría de Ciencia y Técnica de la Universidad de Buenos Aires (UBACYTF602).Director:SusanaRomanosdeTiratel.Colaboradores:AlejandroE.Parada,N ora C. López, Clau dio R. Wuhsagk., Ma ría Ale jan dra T. Pla za y Gra cie la M. Giun ti.

4 Indicadoresdelaactividaddeinvestigaciónaplicadosalasrevistasargentinas. Proyectodeinves tigaciónaprobado y subsidiado por la Secretaría de Ciencia y Técnica de la Universidad de Buenos Aires (UBACYTF602).Director:SusanaRomanosdeTiratel.Colaboradores:AlejandroE.Parada, N ora C. López, Clau dio R. Wuhsagk., Ma ría Ale jan dra T. Pla za y Gra cie la M. Giun ti. 
Information); el debate sobre el uso del idioma español o portugués; el establecimien to de pa rá me tros váli dos y acep ta dos por to dos para evaluar la calidad delas pur blicaciones; elproblemadel nú me ro elevadode títulosencada especialidad, ylaoscilacióncon tinuaentredependenciaein dependenciadelospaísescen tralesodifu sión endogámica delos resul tados deinvestigación. ${ }^{5}$ Los au to res de los tra bajos so bre estos te mas juegan roles diversos en elciclode trans fe renciadelain formación (pueden serproductores, editorescientíficos, evaluado res, publicadores, funcionariosadministrativos de centros de investigación, bibliógrafos, bibliotecólogos o consumidores) y, porlo tan to, persiguen obje tivos quela mayo ría delas ve cesco lisionanentresí o abordan las cues tio nes des dela pro pia ex pe riencia ola apre cia ción personal.Esto no significa desvalorizar esos aportes sino, más bien, ubicarlos dentro de una perspectivamásajustada.

Elpropósitodeestainves tiga ción es conocerquétítulos derevis tas argen tinasespecializadas enhumanida desyciencias sociales estánanalizados, en québases ded atos y en qué nivel(selec tivoo ex haus tivo); es decir, de terminar, en el caso particular deeste artículo, cuáles sonlas tasas derepresenta tividadyde visibilidad dela producciónnacional. Estopermi tirá cono cerqué títu los habrá queindexarybrinda rá da tos exactos y precisos para planificar e implementar efectivamente la estructura bibliográ fica delas hu mani da des y las cien cias so cia les en la Argen tina, y el conocimien to generado contribuirá a constituir acuerdos cooperativos nacionales, regionales e

5 Véa se al res pec to: Cetto, Ana Ma ría y Kai-IngeHille rud, comp.1995. Publica ciones científicasen AméricaLa tina $=$ Scien tificjournalsinLa tin America.1a.ed. Pa rís: InternationalCoun cil of Scienti fic Unions. 305 p.; Fuen ma yor P., Ab del M. 1995. "In ves ti gar y pu bli car”, en Interciencia. Vol. 20, no. 1, 40-46.<http://www.interciencia.org/v20_01/ensayo02.htm> [Consulta: 23 junio 2003]; Krauskopf, Ma nuel y Ma ría Inés Vera. 1995. "Las re vis tas la ti no ame ri ca nas de co rrien te principal: indicadores y estrategias para su consolidación”, en Interciencia. Vol. 20, no. 3, 144-148. <http://www.in terciencia.org/v20_03/art05/index.html> [Consul ta:23junio2003];Armendá riz Sán chez, Saúly Ma. Mag da le na Ordóñez Alon so. 1998. "Las re vis tas aca démi cas de Histo ria en Hispanoamérica: un punto de vista”, en Clio. No. 3. <http://clio.rediris.es/articulos/las_revistas_academicas_de_histo.htm> [Consulta: 22 mayo 2003]; Cetto, Ana María y Octavio Alonso, comp. 1999. Publicacionescientíficasen AméricaLatina= Scien ti fic jour nals in La tin Ame ri ca.1a. ed. París:International Council of Scien ti fic Unions. 489 p.; Ta ller de Ob ten ción de in di ca do res bibliométricos y de actividad científica. 2000. (2: 1-3 de marzo de 2000: Madrid). Relatorías. $<$ http://www.ricyt.edu.ar/Biblioteca/Documentos/2brelatorias.pdf $>$ [Consultado: 23 junio 2003]; TallerdeObtencióndeindicadores bibliométricosydeactividadcientífica. 2003. ( $3^{\circ}: 3-5$ de mar zo de 2003: Ma drid). < http:/ / www.ricyt.edu.ar> [Con sul ta do: 23 ju nio 2003]; Graças Tar gino, Ma ria das y Joa na Coe li Ri bei ro Gar cia. 2000, en Ciência daInformação. Vol. 29, no. 1, 103-117; Haupt, Cecilia.2000. "La 8 Re unión so brelas Re vis tas Aca démicas y de In ves tiga ción. Las re vistascien tíficasla tino americanas: su difu sión y accesoa tra vés debases de da tos”, en Bibliotecauniver sitaria. Nueva épo ca, Vol. 3, no. 2,122-127.

<http://www.dgbiblio.unam.mx/servicios/dgb/publicdgb/bole/fulltext/volIII2/octava.pdf> [Con sul ta: 23 ju nio 2003]; Ratto de Sala, Ma ría Cris ti na y Ama lia Bea triz De lla mea. 2001. "Di fursión, accesoy visibilidaddepublica ciones cien tíficas se ria das deIberoamérica. El sistema La tindex", en Dominguezia. Vol. 17, no. 1.

$<$ http://dominguezia.org.ar/volumen/articulos/17-5.pdf> [Con sul ta: 23 ju nio 2003]. 
internacionalesparaalcanzarla exhaus tividadenelanálisisdelaproducciónespecializadaenambasáreas.

\section{CONTEXTO TEÓRICO}

Michael Keresztesi sostiene que la edad de la información urge a la profesión a concentrarse en el contenidopara maximizarla utilidad so cial de las biblio te cas, de estemodo, no esirrazo na ble argumen tarqueen la sociedad del conocimien tola rela cióndel biblio te ca rio conlos re gis tros estáde termina da, en granmedida, porla na turaleza del aparato informativo que contienen las bibliotecas. El autor separa claramente los roles que juegan estudiosos, científicos y especialistas, de aquellos que representanlosbiblio tecarios en sus rela ciones con elconocimien to.Las de estosúltimos son sinópticas, indirectas y externas, y por tanto afectan el acceso al conocimientoperonoincidenen suprofundidad, suestructuray susus tancia; estoúltimoes dominio de los investigadores. Desde esta perspectiva, bibliotecarios y científicos son, verdaderamente, compa ñe ros enuna em pre sa con junta. Elconocimien toacur mulado de una disciplina reside colectivamente en su literatura; así, la información contenida en esa literatura es una unidad cognitiva básica y no conocimiento en sí mismoporqueésteseconstruyemedian te agrupa mien tos temáticos deinformación relevante a través de operaciones intelectuales complejas que conducen a formulacionesconvincentesyverificables.Simultáneamenteal procesodescritoemergeuna actividadintelectual cuyo propósitonoes produ cirformula ciones válidas, sino, por el contrario, aislar y extractar datos de una literatura de agrupamientos de informaciónhomogéneosy, subsecuentemente,formatearyenvasaresos datosen respuesta anecesidades deinformaciónespecíficas. Talconcepcióndelabibliografíaevidencia su anclaje en la literatura especializada y su vinculación con los procesos de vida de una dis ciplinaparticular, ysu na tu ralezaqueda de termina da, en gran medida, por el niveldemadurezalcanzadoporeláreaestablecida. ${ }^{6}$

Keresz te side finealas disciplinas comoconocimien to queproduceydifunde sistemas, don delainves tiga ción surge comola ac tividad cen tral, pero no pue de ocurrir ais la da de las fuerzas que in flu yen so bre la ejecu ción dela ta rea dein da ga ción. La biblio gra fía, porlo tan to, debe con siderarauna dis ciplina comoun sis te ma de vínculos con mu chos niveles, den tro de la cual cada ac ción produc tivay de apo yo en gen dralos efectoscorrespondientesenlaliteratura dela disciplinayenelmecanismoin formativo que provee el acceso a ésta. Las interrelaciones entre investigación y bibliografía se vuelven ob vias cuan do seexa minanlas dis ciplinas cien tíficas en una ma triz evolutiva. Así, se identifican tres estadios en el crecimiento de un área: el estadio pionero, el de

6 M. Keresztesi, 1982. "The Science of Bibliography: Theoretical implications for Bibliographic Instruction" en Oberman, C. y Strauch, K. Theories of Bibliographic Education. New York: R. R. Bowker, 1982, pp. 3-6. 
elaboraciónyproliferaciónyelesta dio deles table cimien to; además, setipifica la li teraturaquecaracterizaacadaestadio. ${ }^{7}$

Eneltempranoestadiopionerolosprofesionalesysucomunicación con formanun "colegioinvisible" sines tructuraformalniliteratura.Losprimerosadherentespueden serseguidores de unin nova dor oun núcleodeex perimenta do res que sesepa ran de un área establecida. Los registros escritos pueden ser sólo la correspondencia personal, manuscritos, informesinternos, minutasoactasinformales. Es difícilob teneraccesoa unaredsemejante sin un contactopersonal.Elesta diosiguien te de ela bora ciónyproliferaciónproduceuncuerpocrecientedepublicacionesydefuen tes de acceso secundariasquesonrecolecta dasy puestas a dispo sicióndela comunidadporlas bibliotecas. El es ta diodees tablecimien to marcalain te graciónaca démicadeuna disciplinayla disponibilidad de fuentes estándar, tales como enciclopedias, diccionarios terminológicos, libros detexto, manuales, revisiones bibliográficas anuales, etcétera.Deestospatrones de evolución de las disciplinas científicas se derivan tres generalizaciones. En primer lugarexis teuna conexiónin trínsecaen trelabibliogra fía, elapa ra to delain formacióny los procesosinternos de las disciplinas. En segundo términohay una relación directa en treelniveldemadurezdeunadisciplinayelniveldeso fisticación delas herramientas bibliográficas y de referencia. Por último se dan patrones recurrentes y regularidades quepuedenllevaraformulacionesteóricas. ${ }^{8}$

Keresztesi traza la topografía de las disciplinas científicas cuando identifica sus cuatrodimensiones:epistemológica,sociológica,históricaybibliográfica.La dimensiónepistemológicaesla es tructura, elcon tenidoylametodologíadeladisciplina. Se puedenidentificarcincocomponentesdelaes truc tu radeunadisciplina:lamateria y los conceptos; los símbolos lingüísticos (verbales, numéricos o no discursivos); el método de indagación; los valores (actitudes, ética y estética); y la relación del área con otras disciplinas. La dimensión sociológica incluye la organización interna y el esta tus y el apoyo so cial.La mayo ría delas dis ciplinasman tiene una es truc turaprofesional a través de asociaciones y normas que se institucionalizan mediante academias, institutos y el Estado. El estatusse evidenciafinancieramenteporsubsidios y salarios; socialmente por su lugar académico o profesional, y epistemológicamente por el control y uso de su investigación. Los antecedentes históricos de un área del conocimiento, especialmentelascontribucionesclaveylos tex tosclásicos, sonamenudo relevantes para la investigación. También intere sa eles ta dio de de sa rrolloactualomadurez. ${ }^{9}$

Un área primaria de interés en la dimensión bibliográfica son los pro ce sos de investigación, comunicación y difusión del conocimiento jun to con el ori gen, la can tidadyla dis tribución delalitera tu ra es pe cializa da porformatos. Elas pectomás crítico es el apa ra to de la in forma ción, al gu nos delos as pec tos cla ve son cómo sa tis fa celas

7 M. Keresztesi, Op. cit., p. 8-13.

8 M. Keresztesi, Op. cit., p. 13-20.

9 M. Keresztesi, Op. cit., p. 21-23. 
necesida des dela dis ciplina, la es truc tu ra yel nivel de so fis ti ca ción del ac ceso bi bliográfico, las capacidades de las herramientas de referencia, el estado del alfabetismo bibliográ fico,ylas con sidera ciones políticas ydeservicio enlos planeslocales, na cionales. La explora ción dela dimensiónbibliográ ficaes un casoes pecialporquegenera elconocimientodelas manifestacionestopográficasodesuperficie.Latopografía es eldo minio de la biblio gra fía; el he cho de que no sea cen tral para losin te re ses delcientífico no significa que carezca de su propia dignidad, unidad e integridad. Por otra parte, el es tu dio de la to po gra fía de las dis cipli nas pue devolver se el co ra zón dela biblio gra fía cuan do se asu ma que es par te de su pa tri mo nio y que le da su sus tan cia temática, su profundidad y su interioridad. En un sentido profundo, entonces, la bibliografía puede concebirse como un conocimiento sobre el conocimiento, una metacienciaquetieneunamisiónespecífica. ${ }^{10}$

Elpropósitodeestapormenorizada exposicióndelmarcoteó ricoes con tex tualizar lainves tiga ción em pren dida, porque se pien sa que uno delos pro ble mas más agu dos delos es tu dios es ta dís ticos en el cam podela pro duc ción biblio grá fica es ca re cer deuna teo ríasus ten tan tequepermi tain terpre tarlos da tos reco pilados, porloquee $n$ mu chos ca sos se con vier ten en me ras des crip cio nes que no ex plican las cau sas nilas consecuenciasdelosfenómenosestudiados.

\section{METODOLOGÍA}

En primer lugar es importante destacar que este artículo presenta los primeros resultados provisionales de una investigación de mayor aliento y que tiene tres límites fun da mentales: uno dis cipli narporque se cen tra enlas huma nida des ylas ciencias sociales, otro espacial porque estudia la producción argentina, y el otro documentalporque sola menteindaga quépartedela producción plas ma da enartículosde publicacionesperiódicas está indexada en bases de da tosin terna cionales plu ridisciplina rias. La me to do lo gía se en cua dra den tro dela nue va ta xo no mía para los mé todos de investigación social establecidapor Tom Wilson. ${ }^{11}$ Den tro de este en cua dre teórico-meto dológico, elmétodoutiliza doporlainvestigaciónesla ob servación indirec tacones truc turaim puesta. Lainves tiga ción es de tipo ex plora to ria, descriptiva y no experimental.Launidad deanálisisesla revis ta argen tina es pecializada enhumanidades y ciencias sociales; los atributos de la matriz de datos son la representatividad, la visibilidad, el trasla pa mien to y la va can cia en ba ses de da tos in terna cio nales. En este ar tículosólo se pre sen ta ránlos valores dela repre sen ta ti vidad y delavi sibilidad denues trauni dad de análisis en ba ses deda tos mul tidis ciplina rias in terna cionalesyregionales(latinoamericanas).

10 M. Keresztesi, Op. cit., p. 23-24.

11 Tom Wilson, 'Information science' and research methods. http://informationr.net/tdw/publ/papers/slovak02.html [Con sul ta do: sep tiem bre de 2002] 
Las fuen tes emplea das para re colec tarlos da tos se ránlas lis tas de revis tas ana liza das en los servicios de in di za ción y los re sú menes bajo es cru tinio. Para de finirla poblacióneiden tificarcertera men telos títulos serecurrióalos direc to riosoalasbibliografíasdeperiódicas (videinfra, "Procedimientos").

Los procedimientos aplicados pueden responder con exactitud a la pregunta: ¿Cuáles son las tasasderepresenta tividadydevisibilidaddelaproducciónhemerográficaargentina es pecializa daen humanidades yciencias socialesenlas basesde datosbibliográficosinternacionalesyregionalespluridisciplinarias?

Esimportantecon tex tualizaraestees tudiodentrodelabibliogra fíaporquenacey se de sa rro lla a par tir de la preo cu pa ción res pec to de la fal ta de con trol bi blio grá fico de la producción argentina en humanidades y en ciencias sociales. Un presupuesto sub ya cen te es que "[...] com pe te a cada uno de los paí ses el con trolex haus tivo de la literatu raderiva dadelas actividades deinves tiga ción cons tituyén do se asílabbliografía nacional especializada de cada país[...]". ${ }^{12}$ No es ésta una presunción antojadiza sino que re co no ce su origen en la his to ria mis ma delas publica cio nes cien tíficas en los países más desarrollados dado que, en 1665 y con una diferencia de tres meses, aparecen las dos prime ras re vis tas eru ditas-JournaldesSçavants y Philosophicaltransactions - que instauran un modelo de comunicación seguido por el resto de Europa. Poco más de cien años des pués (1778) y antela ne ce si dad de aler tar so bre los avan ces y des cu brimien tos de sus pa res na cio nales y ex tran je ros, se com pila en Ale maniala primera revis taderesú menes es pe cializada, obra deLo renzvon Crellque se tradujo alinglés en 1791. Muy pron to, esta ta rea se pre sen ta comoim po sible para un solo individuoysonlassociedadespro fe sionalesylasacademiasquienesasumenelproceso de organización y difusión considerándolo como uno de sus deberes básicos. ${ }^{13}$ Sin embargo, este proce so no siguelas mis mas eta pas enla Argen tina, don de, comoya se ha men cio na do, sólo se re gis tra un es fuerzo sos te ni do que cu brióla pro duc ción en ArtesyLite ratu raen tre 1959 y 1971 bajola di rección de Augus to Raúl Cortazarycon elapoyodelFondoNacionaldelas Artes. ${ }^{14}$

Ahora bien, se puede suponer que los servicios de indización y resúmenes de alcance internacional controlan, analizan y difunden la producción total de los países que abarcan, lo que haría innecesario que en cada lugar se hagan índices nacionales. Con firmaronoeste supues to con datos fiablesesunodelos objetivosperseguidospor este es tudioque, en primerlugar, determinólosporcentajesderepresenta tividady de visibilidad de las revistas argentinas especializadas en humanidades y ciencias sociales en bases de datos internacionales pluridisciplinarias y que, posteriormente, tomará cada materia replicandoelmodeloyaprobado en las bases de da tosinternacionales unidisciplinarias.

12 Su sa na Ro ma nos de Ti ra tel, 2000. Guiadefuentesdeinformación especializadas: Humanidadesy Ciencias Sociales. 2a. ed. Bue nos Ai res: GREBYD. 281 p.

13 B. Houghton, Op. cit., pp. 81-82.

14 Cfr. Bibliografía argentina de Artes y Letras. No 1 (ene.-mar. 1959)-52 (jul.-dic. 1971). 


\section{PRECISIONES CONCEPTUALES}

Uno de los prime ros pro ble mas que se plan tea ron fue el con cep to de revis ta académica, científica,especializadaodeinvestigación.Encastellanonoexistenpalabras dis tintas paradeno minara este tipo de pe rió dicay diferen ciarlodelas revis tas deinterés general o de los boletines de noticias. El inglés tie ne ma ti ces más ri cos para este universo;así, discriminaentre journal, magazine ynewsletter.

Se consultó una obra dedicada a las publicaciones seriadas que, citando al ALA Glossary ofLibraryandInformation Science de 1983, de fine journalcomo una pe rió dica, es pecialmente una que contiene artículos eruditos y/o difunde información en curso sobre investigación y desarrollo en un campo temático particular. Más adelante, el autor enumera las características de las revistas científicas: los autores son expertos que es criben en un es tiloerudi to para una au dienciaes pe cializa da so bretemas deinves tiga ción; suelentenerunaspecto físicoserio, notasalpiede páginaybibliografías, unco mitéedi torial, pue dencontarconevaluadores ex ternos yestarcubiertas poríndices especializados por materias, y tienen una circulación y tiradas limitadas. ${ }^{15} \mathrm{~A}$ esto se puede agregar el tipo de publicador: universidades, asociaciones y colegios profesionales, academias, institutos o centros de investigación y editoriales comercialesdedicadasalapublicacióncientífica.

Con este concep to rec tor se podrían des car tar los bole tines de no ti cias onewsletters, que son publicaciones se ria das forma das por una ounas po cas ho jas im pre sas que contienennoticiasoinformacióndeinterés, principalmenteparaungrupoespecializado. ${ }^{16}$

Ade más, sede ci dióno in cluirlas se ries mono grá fic cas nilas re vis tas pu blica das después de 1997, porque es al ta men teim pro ba blequeés tas ya hubie ran sidoin clui das en las ba ses de da tos in terna cionales, dado que un prin ci pio ge ne ral para que esto su ce da esquelarevis ta demues treuna con tinuidadenlapublicacióndealmenostresaños.

Laimportan cia de estere corte previoes tribó enlanecesidaddetenermuyenclaro el tipodere vis tas quein te gra ríanlapoblación depublicaciones periódicasargentinas es pe cializadasenhumanidades y cien cias socialesparaconfrontarconlas lis tasindexadasporlosserviciosdeindizacióny resúmenes examinados.

Otros as pectos conceptuales quesehanusa doenestees tu dioson losindicado res derepresentatividadyvisibilidad,loscualessehandefinidoasí:

- Indicadoresde representatividad: es el porcen ta je de títu los de un país en rela ción con la lista total de títulos analizados en una base de datos.

$$
I R=\frac{\text { títulos de unpaís en } B \text { de } d}{\text { número total de títulos en } B \text { de } d} \quad \times 100
$$

15 T. Nisonger, Op. cit., p. 4-5.

16 T. Nisonger, Op. cit., p. 6. 
Indicadores de visibilidad: es la relación entre la producción total de un país en una temática determinada y el número de títulos indexados en las bases de datos internacionales.

$$
\text { Vi }=\frac{\text { títulos deunpaísen } B \text { de } d}{\text { númerototaldetítulos dela disciplina }} \times 100
$$

\section{PROCEDIMIENTOS}

Enprimertérmino, se establecióunalista de se ria das es pecializa das en lasáreascubiertasutilizan do como fuenteelBINPAR, ${ }^{17}$ compila doporelCentro ArgentinodeInformación Científica y Tecnológica. De ese grupo inicial de cerca de 1,000 títulos, se hizo un primerre corteeliminan dolas series monográ ficas, las revis tas cuyoes ta do era des conocido, las ce sadas y laspublica das des pués de 1997, conlo queselle góa unalista algo supe rioralos 500 títulos. Tenien do encuentaloscri te rios pre viamen te es tablecidosrespectodelos publicadoresyeltipodematerialincluido, sedescarta ronlosboletines denoticias, lasrevis tas dedatosestadís ticos, deindicadoreseconómicos, bbliográficasodereseñasyseob tuvounalistaprovisionalde 356títulos, querepresentatan to la pobla ción como la mues tra. De todos modos se usó estenú me ro paralos pri me ros cál culosqueseafinaráncuandoselleguealalistadefinitiva.

En segundo lu gar se divi die ron las ba ses de da tos biblio grá fícos-servicios deín dices y resúmenes-en:a)latinoamericanasmultidisciplinarias,b)internacionales mul tidisciplinariasyc)internacionalesunidisciplinarias.Seanalizaronlaslistasderevistasindexadas ex trayendolos títulosargentinos ydel res tode AméricaLatina,yse es tudiaron las características fundamentales delas bases, so bretodolore feridoa ex haus tividady alcance cronológico, dado que sibien algu nas bases in clu yen ciertas re vis tas comoindexadas, en la ac tua lidad han de ja do de ha cerlo. Eneste artículo sólo se pre sen tanlos resultadosinicialesyprovisionalesreferidosalosantedichospuntosa)yc).

Finalmentesesacaronlosporcentajescorrespondientesala representatividadde laproducciónla tinoame ricanaencadauna delas basesanalizadas, ylos de visibilidad de la producción argentina. Para esto y para establecer futuras relaciones se diseñó una base de da tos rela cionalenMicro soft Access ${ }^{\circledR}$ don de, para cada título de revis ta argentinase es tablecieronlos siguientes campos:título, ISSN, periodicidad,lugarde edición, editor, tipo de en tidad edito ra, fecha, ca te go ría te má tica, materia1, ma teria 2 , his to riay, además, paracada título siestáin dexado, en québase/s, o sinoloestá.

17 BINPAR [recursos electrónicos] : Bibliografía Nacional de Publicaciones Periódicas Argentinas Re gis tra das. 2000- . Bue nos Ai res:CAICYT, 2000- . 1 CD-Rom. ISSN: 1515-7040. 


\section{RESULTADOS INICIALES}

\section{Bases de datos multidisciplinarias de alcance latinoamericano o hispanoamericano ${ }^{18}$}

Handbook of Latin American Studies: HLAS Online

Handbook ofLatin American Studies: HLAS Online. [Si tio Web]. Com piled by the His panic Division, The Library of Congress. <URL:http://lcweb2.loc.gov/hlas>. [Consultado: junio 2001].

Se tra ta de una bi bliogra fía se lec tiva so bre América La tina, dado que más de 130 especialis tas de todoel mundoeligenal rededorde 5,000 trabajos parasuin clu siónen unvolu men delHandbook. Se publicasininterrupciones des de 1939 ylabase de da tos enlíneain cluyelosregis tros desde esa fe cha.Losporcen tajes delos forma tosanalizados son del $60 \%$ paralibros y del $40 \%$ para publica cio nes perió dicas. Serevisólalis ta derevis tasindexadas enlos volú menes ac tuales 50-59, quepresen taun to talde2.685 títu los, 1192 de los cuales son la ti no ame ri ca nos (44\%), y den tro de este gru po se incluyen165títulosargentinos $(6 \%)$.
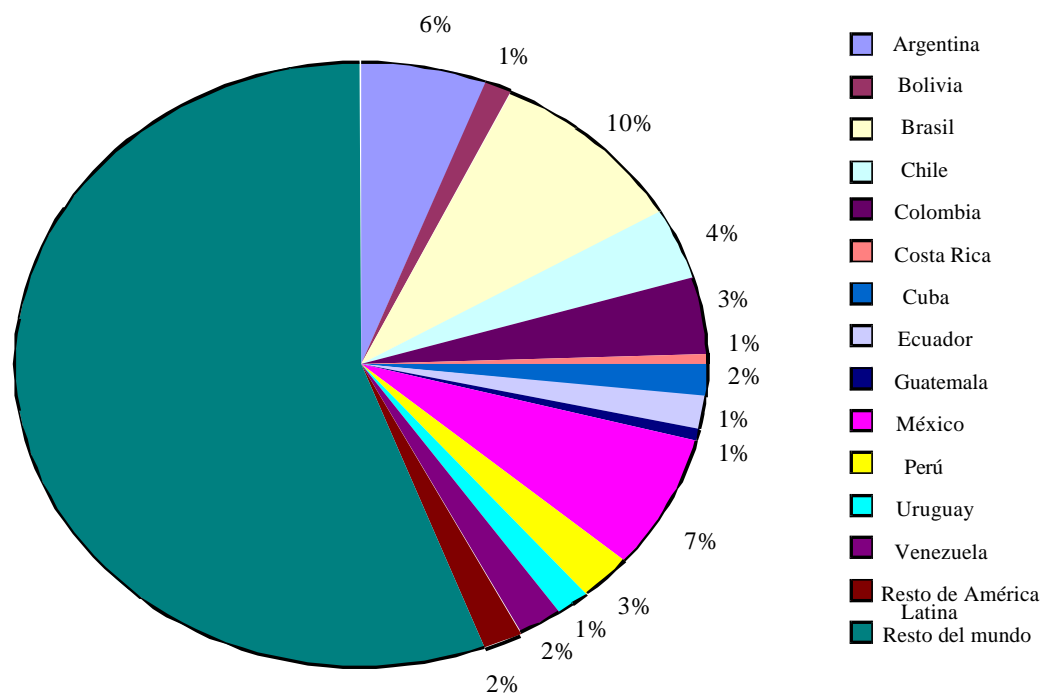

18 No se hain clui do el La tin dex-Sis te ma Regio nal de In for ma ción en Línea para Re vis tas Cien tíffcas de América La tina, el Ca ribe, Es pañay Por tu gal por que has ta la úl ti ma fe cha decon sul ta (noviembre de 2003) no ha bía ini cia do lain de xa ción analític ca de los con te ni dos de las publicaciones periódicasqueregis tra. 
En elres to de AméricaLa tina, con menos del1\% seubicanHon duras, Nica ra gua, Panamá, Paraguay y República Dominicana. Se desea volver a insistir en que esta base de datos no indexa exhaustivamente los títulos incluidos sino que selecciona aque llos ele gi dos por los ex per tos. Con esta salve dad, si to ma mos en cuen ta el to tal de revistas argentinas especializadas en humanidades y ciencias sociales, veremos que en elHandbookestávisible, selectivamente,el46\% delaproducciónnacional.

\section{HAPI Online}

HAPI Online. [Si tio Web]. Pro du ced by an edi to rial staff at the UCLA La tin Ame rican Center.

<URL: http://gseis.ucla.edu/hapi/html> [Consultado: julio 2001].

El Hispanic AmericanPeriodicalsIndexes una base de da tos don de la ma yo ría de la indización inicial está hecha por bibliotecarios y profesores latinoamericanistas que trabajan voluntariamente. Se publica desde 1970. Sólo analiza revistas y lo hace en pro fun di dad. La base pre sen ta una sola lis ta de pe rió di cas don dein cluye tan tolos tí tulos queindexó en elpasadocomolosqueanalizaac tualmente. Se trabajóconlalis ta en curso que incluye 228 ítems. América Latina está representada con 135 títulos (59\%) y den tro de este gru pola Argen tina al can za un 4\% (9 pe rió di cas). En elHAPI, cuan do se tiene en con si de ra ción el to tal de re vis tas argen ti nas de lasáreas es tu diadas, estárepresentadosóloel3\% delaproducciónnacional.

\section{Representatividad de la producción latinoamericana en el HAPI}
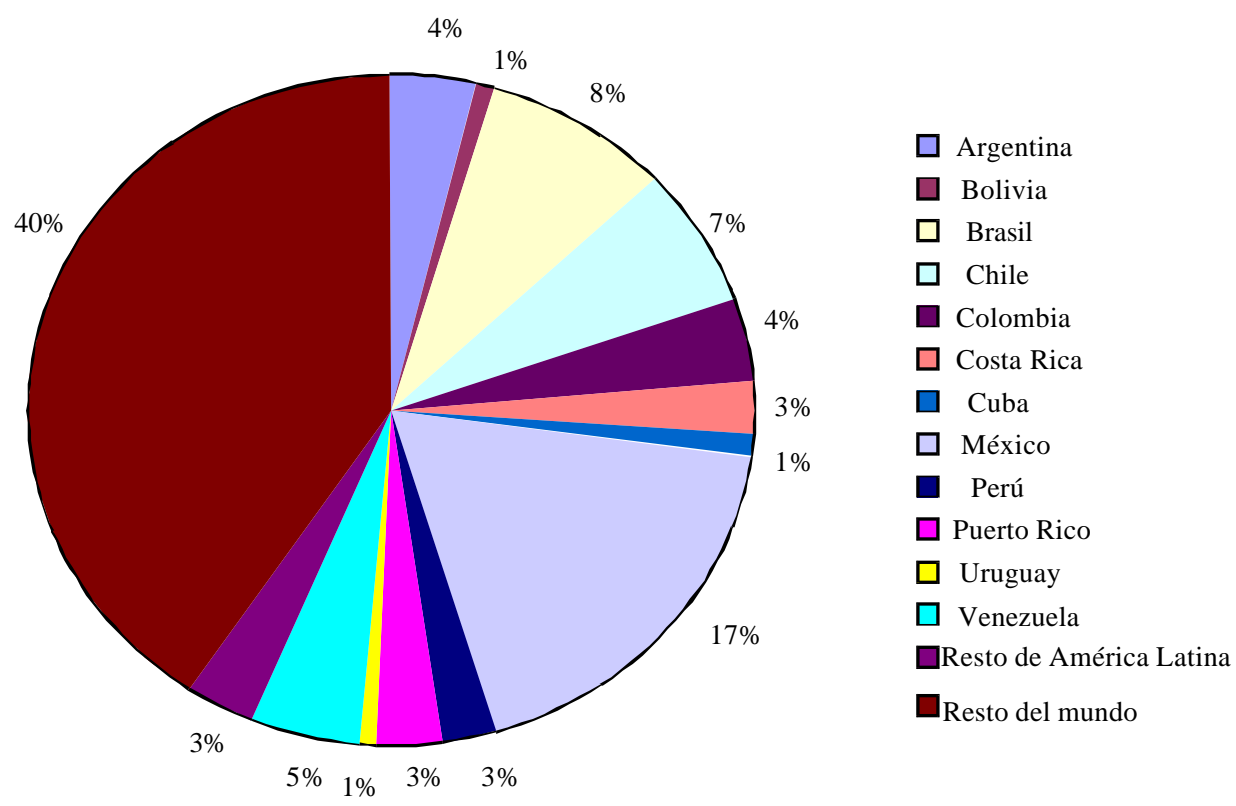


\section{Bases de datos multidisciplinarias de alcance internacional}

Social Sciences Citation Index y Arts $\mathbb{E}$ Humanities Citation Index

Las dos bases de datos del Institute for Scientific and Information Science (ISI) útiles para este es tu dio son el SocialSciencesCitationIndexcon 1755 títulos ana lizadosy el Arts \& Humanities CitationIn dex con 1126. En las áreas de hu mani da des y cien cias so ciales, América La tina estámuy esca samentere pre senta da con un $0,4 \%$. En el primercaso sólo tres paí ses: Bra sil (1 títu lo; $0,1 \%$ ), Chile y Mé xi co (2 títu los y $0,2 \%$ cada uno). Como se ve, la Argentina tiene una representatividad nula. En el caso de las cienciassociales, sucedecasilomismo.Seincluyen: Argentina,Brasil,ChileyColombia (1 título y $0,06 \%$ cada uno) y México ( 2 títulos; $0,1 \%$ ). En el gráfico mostrado abajo se han su ma do am bas ba ses (2.883 pe rió di cas), lo que hace quela tasa de in clusiónderevistasargentinasseade $0,03 \%$.

Si,porotraparte, se con sidera el to tal de re vistas argen tinas delasáreas es tudiadas en re la ción con las in clui das en las ba ses delISI, está re pre sen ta do sólo el 0,3\% dela producciónnacional.

\section{Representatividad de la producción latinoamericana en $\mathrm{AHCl} \& \mathrm{SSCl}$}

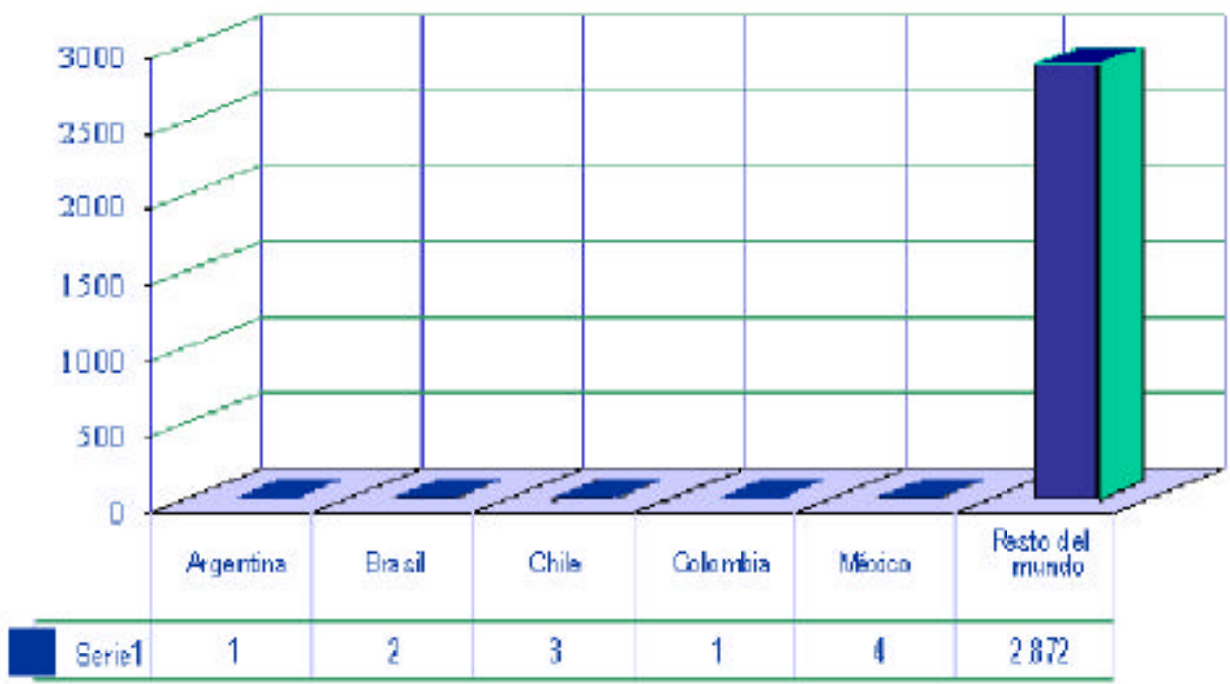




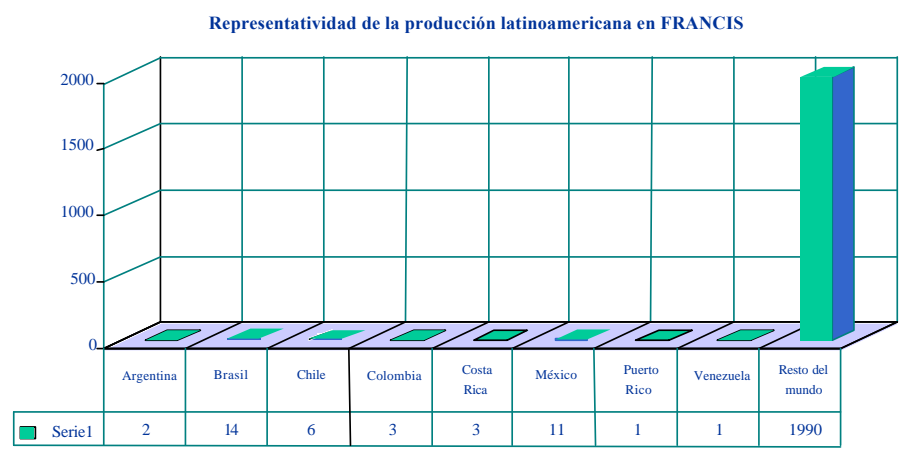

Francis

Francis es un archivoinformático disponible en Internet en el sitiodelIns titutde l'Information Scientifique et Technique, <URL: http//www.inist.fr>. Compilado por el Centre de Documentation en Sciences Humaines (CNRS), reemplaza desde 1995 alconocido Bulletinsignalétique en soportedepapeleincluye tan to hu manidades como cien cias so ciales. Elto tal de pe rió di cas in de xa das es de 2033. La si tua ción mejora algo respecto del caso anterior, dado que América Latina alcanza un 2\% y hay más países representa dos: Argentina, PuertoRico y Venezuela (2 títulosy0,1\% cada uno), Bra sil (14 tí tu los;0,7\%), Chile (6 títulos;0,3\%). Colombia y Cos ta Rica (3 títulos y0,15\% cada uno), México (11 títulos;0,5\%). EnFRANCIS, sise tiene enconsideración el to talde revis tas argen ti nas delas áreas es tu dia das, está re pre sen ta dosólo el 0,6\% delaproducciónnacional.

IBZ

IBZ:Internationale Bibliographie der Geistes-und Sozialwissens chaftlichen Zeitschriftenliteratur $=$ IBZ : International Bibliography of PeriodicalLiterature in the Fields of Arts and Humanities and the Social Sciences. [Sitio Web]. Dietrich Verlag.

<URL: http:/ /www.dietrich-bibliogr.de>.

La IBZes un serviciodein diza ciónale mán que se com pila des de 1896 y proveeaccesoa uncuerpoim portantedelite ra turape riódica, median te elanálisis de 4650títulos. Elporcen taje del 1,5 (69 títulos) dela pro duc ciónla ti no ame rica na en este re perto rio es si mi laralde FRANCIS. Así, hay revis tas de Argentinay Vene zuela (4 títulosy 0,1\% cada uno), de Bra sil (18 tí tu los;0,4\%), de Chile (7 tí tulos; 0,2\%), de Colombia (13 títu los; 0,3\%), de Cos ta Rica y Puerto Rico (2 títulos y 0,05\%), de Mé xi co (16 títur los; $0,3 \%$, de Ni ca ra gua, Pa na má y Perú ( 1 tí tulo y $0,02 \%$ cada uno). En el $I B Z$, cuandosetieneenconsidera ción el to talde revis tas argen tinas delas áreas es tudia das, está representadosóloel1\%delaproducciónnacional. 


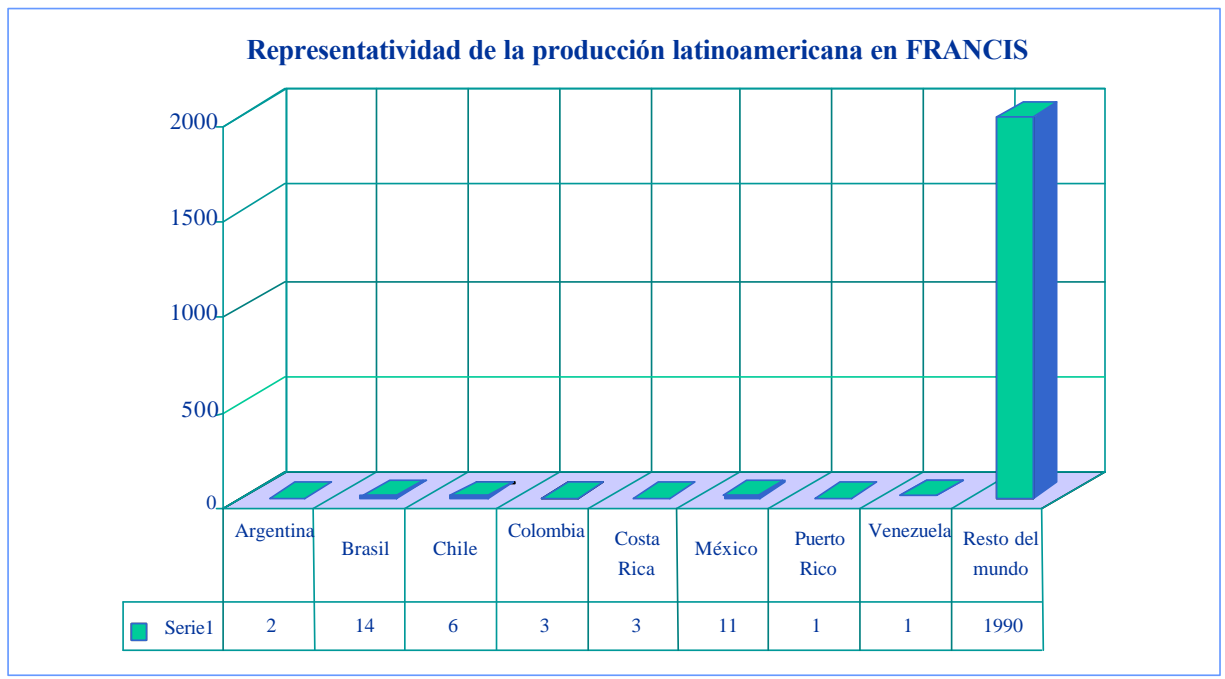

International Bibliography of the Social Sciences

La International Bibliography ofthe Social Scien ces (IBSS) esuna conocidacompilación bibliográfica en el área de las cien cias so cia les que se ini ció en 1952. Pro du ci da en la ac tua li dad por la Lon don School of Eco no mics, su ver sión en línea se lo ca liza en el <URL: http://www.lse.ac.uk/IBSS/>. Analiza en la actualidad 1751 periódicas de todo el mundo. La producción latinoamericana está representada con 32 revistas ( $2 \%$ ) dis tribuidas en Argen tina ( 7 títulos; $0,4 \%$ ), Chile ( 6 títulos; $0,3 \%)$, Colombia (8 títulos; $0,5 \%$ ), Cos ta Rica, Ecua dor, Pa na má, Pa ra guay, Perú y Ve nezuela (1 tí tuloy 0,05\% cadauno),Cuba (2títulos; $0,1 \%$ ) y Puerto Rico (3títulos; $0,2 \%$ ).

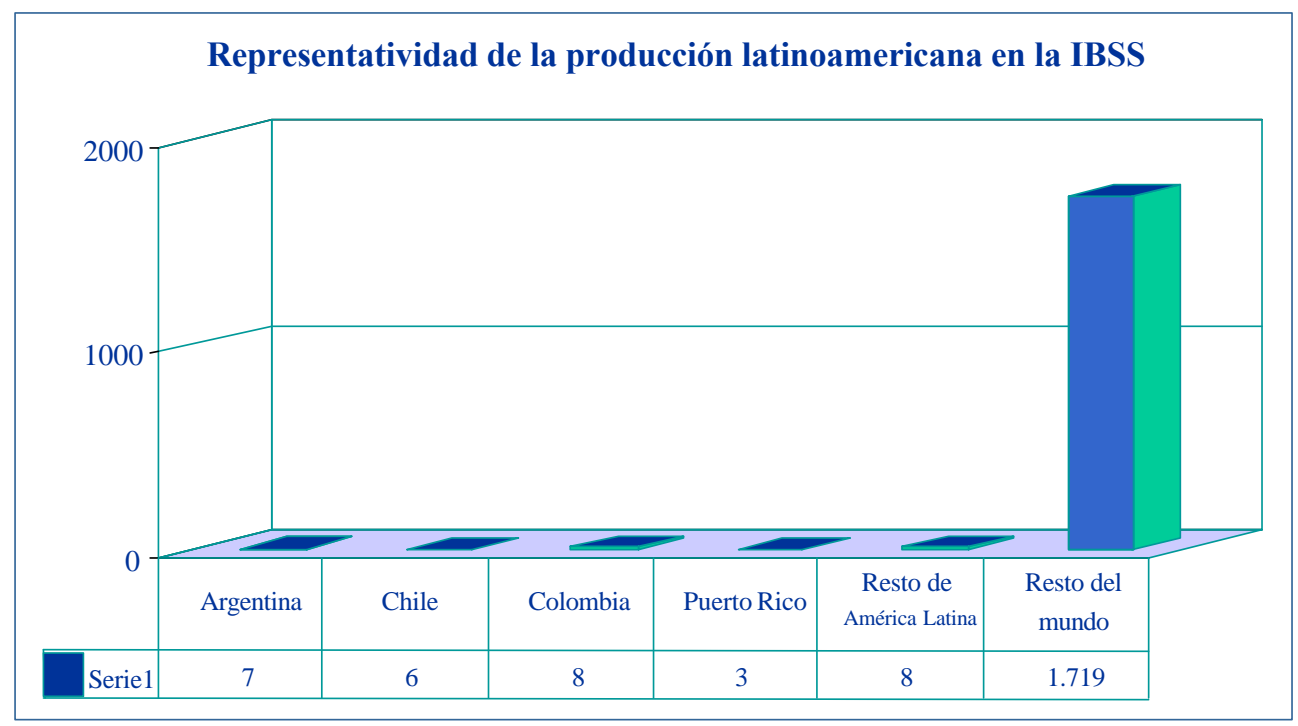


A continuación se presentan dos gráficos y una tabla resumen para la Argentina queilus tranlos porcentajes de repre sen ta tividad argen tinaydelaproducción nacional, to man do como base en este caso 356 tí tu los que reú nenprimafacielas ca rac te rís ticas pre-definidas, en las bases de datos multidisciplinarias. Finalmente, se elaboró unatablaresumendelosdatos recopiladosparaArgentina.
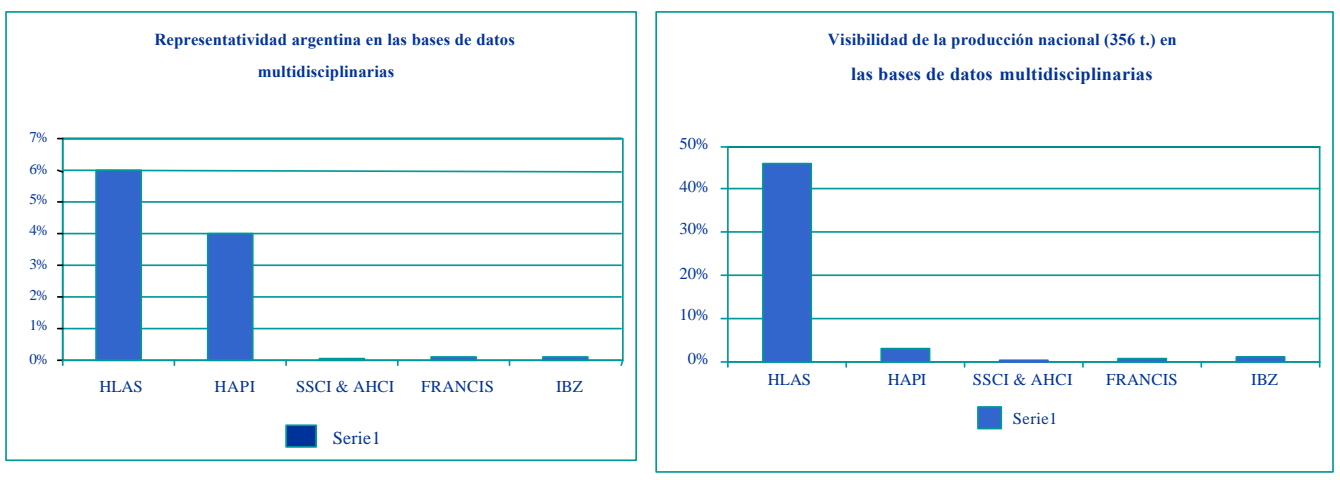

Tabla 1

Resumen de los datos compilados para Argentina

\begin{tabular}{|c|c|c|c|c|}
\hline $\begin{array}{c}\text { Datos } \\
\text { Bases de da tos }\end{array}$ & $\begin{array}{l}N^{\circ} \text { de perió dicas } \\
\text { in dexa dasen las } \\
\text { bases de datos }\end{array}$ & $\begin{array}{l}N^{\circ} \text { de periódicas } \\
\text { argentinasinde- } \\
\text { xa das en las ba- } \\
\text { ses de datos }\end{array}$ & $\begin{array}{l}\text { Representativi- } \\
\text { dad argentinaen } \\
\text { las ba ses de } \\
\text { datos }\end{array}$ & $\begin{array}{c}\text { Visibilidad de la } \\
\text { pro duc ción na cio- } \\
\text { nal ( } 356 \text { t.) en las } \\
\text { ba ses de da tos }\end{array}$ \\
\hline$H L A S$ & 2.865 & 165 & $6 \%$ & $46 \%$ \\
\hline HAPI & 228 & 9 & $4 \%$ & $3 \%$ \\
\hline$S S C I \& A H C I$ & 2.883 & 1 & $0,03 \%$ & $0,3 \%$ \\
\hline FRANCIS & 2.033 & 2 & $0,1 \%$ & $0,6 \%$ \\
\hline IBZ & 4.650 & 4 & $0,1 \%$ & $1 \%$ \\
\hline
\end{tabular}

Lasbasesdedatoslatinoamericanas estudiadas presentandiferencias sustanciales quelas vuelvenin comparables:númerodetítulosindexados, políticasparaelanálisis delas perió dicas-selectividad $v$ sex haus tividad. De to dos mo dos, la ra tio más alta de representatividad tanto de la base de datos como de la producción nacional la proporcio na el Handbook of Latin AmericanStu dies, pero esa tasa no nos in di ca cuán to del totalunitario(artículos)seincluyeenelrepertorio.

Lasbasesdedatosinternacionales multidis ciplina riasmuestrantasas deinclusión yderepresentatividad dela producción nacionaligualmen tebajas, yen esa ten dencia lodes tacan porloacen tuado,los productosdelISI. 


\section{DISCUSIÓN}

Elcaso argentinopresen tauna situa ciónparadójica.Las dis ciplinashumanísticas y de las ciencias sociales alcanzaron, hace ya largo tiempo, su estadio de establecimiento: es tánbienasenta das enlas universidades; seorganizanendepartamentosau tóno mos; han formaliza dorígidamen tesus programaseduca tivos, reque rimien tosy calificaciones; desarrollan curricula estructurados;implementan programas de grado ydepostgrado (doctorados, maes trías, especializa ciones); lainves tiga ción estáin stitucionalizadaencentros, departa men tos, cáte draseins titu tos; exis teunaes tratificación académica; y se obtienen contratos de investigación gubernamentales y privados. Sinembargo, la es tructu ra bibliográfica no secondice coneste es ta dio, dadoque el apa ra to delain for ma ción proporciona ba jos niveles de sa tis fac ción delas nece sidades para las diferentes disciplinas; la estructura y el nivel de acceso bibliográfico dis tanmuchode serso fis tica doso siquie ra me dia na menteaceptables; ylas con sidera ciones políticas y de servicio es tán au sen tes en los pla nes tan to delasins ti tu ciones comodelaNación.

Conlos re sul ta dos provisio nales presen ta dos se pue de afirmarque enla Argentina se investiga y se publica en las áreas consideradas por este estudio,pero también quees al tamenteim probablequelos mis mosargentinosconozcan, recuperenyconsu man el fru to de tan tos es fuerzos hu manos y ma te riales y, mu cho menos aún, lacomunidadespecializadadeotrospaíses.

¿Eslícitoes perarcon fiada mente que otros ha ránel tra ba jo que no so tros no hacemos? Engeneral, los crite rios dein clu sión en las bases deda tosinternacionales es tán pautados por normas cuantificables o binarias; por ejemplo, arbitraje, puntualidad en la frecuencia, distribución internacional, jerarquía científica del editor y de los miembros de los consejos o comités asesores, ti ra da, otras ba ses de da tos que in dexanlas revis tascon sidera dasycumplimien todeestán daresinternacionalesenlap resentación formal de los contenidos. Se presupone así que el cumplimiento de cada una de estas regulaciones dará como resultado contenidos intelectuales de calidad. Sinem bargoen las dis ciplinas que aborda este es tu dio, si se to ma racada unode es tos puntos serían muypocos los títulos selec cionados, y lomás pro bable es quequeda ran afuerapublicacionesperió dicas delargatra dicióny reconocidonivelintelectualpero alas que seles hace di fícil cum plircon la fre cuen cia o tienen políticas edito riales que, sin acatar los usos de la dobleevaluaciónanónima,garantizanunarbitrajeimplícito en comen dán do le a un es pe cialis ta de gran nivella compila ción decadauno delos nút meros, o, sim plemente, des conocen o no pue den aplicarlas normas en uso. Encuantoalrequisitodeestarindexadas porserviciosinternacionales, elcasoseasemeja al de los ban que ros: se le pres ta di ne ro a quien ya lo tie ne, acá se in clu yen tí tu los que otros ya ana lizan, pero ¿cómo se hace para en trar al club por pri me ra vez? Y ¿es ló gi co que siempreaparezcanlasmismasperiódicasendiferentesbasesdedatos? 
Cuandolos bibliotecarios que desempeñansus funciones enuniversidadesycentros es pecializa dos asu manla dimen sión bibliográ fica delas dis cipli nas comouna de sus ta reas fundamenta les y coordinen sus es fuerzos para lograrelcontroldela literaturagenerada porlosinvestiga dores, seconvertiránen copartícipes dela producción delconocimientoyenagentesactivos desudifusión. Seesperaqueestainvestigación ayude a trazarun pa no ra ma dela situa ción y con tribuya valo rarlo quenos pertenece yrepre sen ta. Seacep ta quelas bibliogra fías au men tan su selec tividaden rela ciónc on elnú me roy va rie dad delos do cu men tos que in clu yen y delas ma te rias que abarcan, y también es una re gla que el país res pon sa ble de la com pila ción acen túa la pro pia produc ción. La his to ria del de sa rro llo de la es truc tu ra bi blio grá fica delos países centrales muestra una clara conciencia de la responsabilidad y de una productiva división deltrabajo;losresultadosestánalavista.

Hace ya poco más de vein te años, el holan dés Jo han van Halm se preo cu pa ba por ladependenciain formativaalaqueconsiderabauna partedela dependencia tecnológica, sien do estaúl ti ma sólo un as pec to de una de pen den cia más am plia de la orga nizacióneconómica y edu ca tiva. Elau tor aler ta ba con tra los pe li gros de la con cen tracióndelconocimien toenunos pocospaíses yapelabaalacooperaciónin ternacional para equilibrarlasitua ción. Den trodelas condiciones que po drían prevenirla dependenciain forma tiva, vanHalmpresenta una queresumea to das las demás: es tima ción del equilibrio apropiado entre auto-suficiencia interna e interdependencia internacional; es de cir, la re ducción del porcentaje dein forma ción im por ta da y el fo mento delvalordelapropiain formacióngeneradaquedebehacercadapaís. ${ }^{19}$

\section{REFERENCIAS BIBLIOGRÁFICAS}

ROMANOSDETIRATEL, S. Guíadefuentesdeinformaciónespecializadas: Hu manidades y Ciencias Sociales. 2a. ed. Buenos Aires: GREBYD, 2000.

KERESZTESI, M.1982. “The Scien ce of Biblio graphy: Theo re ticalimplications for Bibliographic Instruction", en: OBERMAN, C. y STRAUCH, K. Theories of Bibliographic Education. New York: R. R. Bowker, 1982.

NISONGER, T. 1998. Management of serials in libraries. Englewood, Colo.: Libraries Unlimited, 1998.

HALM,J.van.1978. "Internationalcooperation ornationaldependence”, en Special libraries, 1978, vol. 69, p. 201-205.

19 J.VAN HALM.1978. “Internationalcooperationornationaldependence”, enSpeciallibraries, 1978, vol. 69, p. 201-205. 